\title{
Building a Smooth Medical Service for Operating Room Using RFID Technologies
}

\author{
Lun-Ping Hung, ${ }^{1}$ Hsin-Ke Lu, ${ }^{2}$ Ching-Sheng Wang, ${ }^{3}$ and Ding-Jung Chiang ${ }^{4}$ \\ ${ }^{1}$ Department of Information Management, National Taipei University of Nursing and Health Sciences, No. 365, \\ Ming-te Road, Peitou District, Taipei 11219, Taiwan \\ ${ }^{2}$ Department of Information Management, SCE, Chinese Culture University, 55, Hwa-Kang Road, Yang-Ming-Shan, \\ Taipei 11114, Taiwan \\ ${ }^{3}$ Department of Computer Science and Information Engineering, Aletheia University, No. 32, Zhenli St., Danshui Dist., \\ New Taipei City 25103, Taiwan \\ ${ }^{4}$ Department of Computer Digital Multimedia Design, Taipei Chengshih University of Science and Technology, \\ No. 2, Xueyuan Rd., Beitou, Taipei 11112, Taiwan \\ Correspondence should be addressed to Ching-Sheng Wang; cswang@mail.au.edu.tw
}

Received 21 January 2014; Accepted 6 May 2014; Published 13 July 2014

Academic Editor: Young-Sik Jeong

Copyright @ 2014 Lun-Ping Hung et al. This is an open access article distributed under the Creative Commons Attribution License, which permits unrestricted use, distribution, and reproduction in any medium, provided the original work is properly cited.

Due to the information technology advancement, the feasibility for the establishment of mobile medical environments has been strengthened. Using RFID to facilitate the tracing of patients' mobile position in hospital has attracted more attentions from researchers due to the demand on advanced features. Traditionally, the management of surgical treatment is generally manually operated and there is no consistent operating procedure for patients transferring among wards, surgery waiting rooms, operating rooms, and recovery rooms, resulting in panicky and urgent transferring work among departments and, thus, leading to delays and errors. In this paper, we propose a new framework using radio frequency identification (RFID) technology for a mobilized surgical process monitoring system. Through the active tag, an application management system used before, during, and after the surgical processes has been proposed. The concept of signal level matrix, SLM, was proposed to accurately identify patients and dynamically track patients' location. By updating patient's information real-time, the preprocessing time needed for various tasks and incomplete transfers among departments can be reduced, the medical resources can be effectively used, unnecessary medical disputes can be reduced, and more comprehensive health care environment can be provided. The feasibility and effectiveness of our proposed system are demonstrated with a number of experimental results.

\section{Introduction}

The competitiveness of today's medical industry lies not only in the pursuit of higher medical technology breakthroughs, but also in the provision of a patient oriented, safe, and high-quality medical environment as the goal. Surgery has always been one of the busiest tasks in hospitals. Negligence of any details during operation is likely to cause permanent damage on patient. Therefore, it is necessary to strengthen the process of monitoring and management inside operating rooms. How to establish a system that can accurately identify patients, track their locations, and be integrated into the medical information system to enhance the medical service quality and efficiency has become an important issue of the development of medical science. In this paper, the radio frequency identification, RFID, was imported into the realtime positioning mechanism for surgical patients during the process of surgery. This mechanism can identify patients automatically and detect the locations of the surgical patients accurately from the time they were transferred from the wards into the surgery waiting rooms, operating rooms, and recovery rooms. Signals of RFID devices were transmitted to the back-end server. It is a surgical patients positioning system used before, during, and after the process of surgery. Through the real-time patient information updates to assist medical personnel's medical and nursing operation, they can 
have more time to deal with various measures for surgery in attempt to reduce the occurrence of errors and to provide surgical patients with safer medical environments and better medical quality.

The proposed mechanism can overcome the existing blind spot during the process of surgery using wireless transmission technology. RFID technology refers to technology that can identify objects through wireless communication, which is made up of the electronic tag, the reader, and the integration system. Through the communication between the tag and the reader, the reader can access the identification data stored in the tag without contacting with the tag or existing within the visible range of the reader, thereby achieving the function of object identification. From the technical point of view, the tag can be divided into passive mode, active mode, and semiactive mode.

At present, technologies using RFID in the research of positioning are divided into four categories: time of arrival (TOA), time difference of arrival (TDOA), received signal strength (RSS), and angle of arrival (AOA). TOA and TDOA are positioning techniques using time as the basis of measurement and they require accurate synchronization and frequency. TDOA measures the distance by using the relative time of the received signal, whereas TOA uses the absolute time to measure the distance. As compared to the basis of time, RSS uses the signal strength as the basis of measurement, but this method is likely to be interfered by multipath and objects, resulting in measurement errors. AOA uses antenna arrays or directional antennae in LOS (Line-ofsight) signal transmission environment to produce accurate results. RSS is the most suitable positioning mode for indoor environment at present, considering cost and accuracy [1-3].

In this paper, the RSS positioning technology has been adopted. In addition, with the signal strength value that decreases as the distance increases, which is a feature of the RSS, the concept of signal level matrix, SLM, was proposed. Moreover, through the implementation of RFID, patients can be accurately identified and the occurrence of surgical errors can be reduced. Using the real-time patient location positioning system, repeated checking of the processes can be reduced and the surgical processes can be more transparent and automated, thus leading to the establishment of the patient-oriented medical service model. In Section 2, we briefly introduce the development of RFID technology and its application on medical practice. The methodology and the application of the proposed mechanism are described in Section 3. Section 4 contains implementation and experimental results. The last section, Section 5, is conclusions.

\section{Related Work}

Technologies for tracking and tracing are plenty [4-6]. RFID is one of them. It is an important technology for tracking objects. One of the most important RFID applications is positioning and tracking of objects inside building due to its capability of high speed contactless identification. Supply Chain companies like Wal-Mart and Tesco have deployed RFID systems in some of their supply chains. In this paper, we propose patient position tracing system based on RFID technology. Regarding current development of researches on RFID technology, RFID technologies used in healthcare industry and the position tracing mechanism for surgical procedure. They are described in this section.

2.1. Current Development of Researches on RFID Technology. In previous researches, many researches on RFID technology were mainly based on passive mode. Backscattering RFID is a type of passive RFID technology employing tags that do not generate their own signals but reflect the received signals back to the readers $[7,8]$.

Papers published in the field of RFID research are numerous. Among them, Systems like SotON, LANDMARK, VIRE, and LEASE [9-12] are very famous in the research field of RFID. SpotON uses GPS technology to the RFID indoor positioning system. It uses received signal strength indication to calculate distance and leads to relevant researches in the RFID positioning method. Then, the LANDMARC system was proposed by Liu et al. This system adopts reference tag as the base of improving positioning accuracy. Due to the improvement of positioning accuracy, RFID positioning method has received more attention. Inspired by the LANDMARC system, more refined RFID positioning systems were proposed. Among them, VIRE system uses virtual reference tag and elimination algorithm to reduce the occurrence of errors and to improve the RFID positioning accuracy. LEMT system adds tree structure judgment method to accelerate the calculation of reference tag and area position.

According to previous researches, the main reason causing errors of positioning system is unstable RSSI value of Tag signal, and it is likely to be interfered by environmental factors. Thus, using only signal strength for positioning often results in large errors [13].

\subsection{RFID Technology Used in Healthcare Industry. RFID is} one of the technologies that potentially contribute to the development of medical study. Currently, RFID technology can overcome the bottleneck of workflow management in the field of medical practice. RFID technology can drastically reduce or entirely eliminate the time medical or nurses spend on patient care [14].

Some researches dedicated themselves to the development of point-of-care data delivery based on RFID technology. Staff badges, medication packaging, and patients' identity bracelets contain RFID technology. This facilitates identification of a patient by caregivers, who are, thus, able to submit orders in real-time at the very point of care, instead of being handwritten and sent off for future input. This kind of system saves time and reduces the chances of human error $[15,16]$. Moreover, RFID technology can be applied on asset tracking and locating. Some position-based indoor tracking systems have been used in hospitals, where expensive equipment needs to be tracked to avoid being stolen and the patients can get guidance to efficiently use limited medical resources inside complex environments of the hospital. For instance, surgical instruments and other devices must be properly cleaned and packaged between uses. 
Tags on the instruments and readers on the sterilization chambers and storage cabinets can validate proper cleaning and help locate needed instruments. RFID can also facilitate better management of medical equipment, medicine, and storage which leads to a more efficient and less medical error environment [17-19].

RFID technology can also be applied on patient location: tracking the location of patients in case of long-term care, mentally challenged patients, and newborns. It has the ability to determine the location of a patient within a hospital and facilitate the delivery of health care. For example, when a patient arrives in a lab for a radiology exam, medical staff is instantly alerted via the RFID tag and the transfer of records can be affected immediately. Patient tracking mechanism can manage patients efficiently to provide a better health care service [20-22].

2.3. The Positioning Mechanism for Surgical Procedure. Hospital risk management mostly deals with the high-risk involved activities performed in the hospital. Several RFID technologies are currently available to reduce the risk. The signal strength of RFID chips can provide information to the tagged subject in addition to a unique identity $[18,23]$. Active and passive RFID devices were used to track medical personnel, patients, medical equipment, and the deposit of blood to enhance the safety of surgical operation [24]. This adds substantial value to the management of tracing the position of surgical patients. The healthcare environment can be characterized by process broken down into tasks. For instance, a surgery can be broken down into several tasks: signing in at the reception, waiting for the physician, and so forth. Thus, monitoring the surgery progress definitely benefits the adjustment of taking care of personnel, medical equipment, and other related medical resources. Patients can be operated in a safe and efficient medical environment.

Applications of RFID technologies are widely spread these days. Except used in medical industry for personnel identification and management, it is used in operation room for identification and positioning. The passive RFID tracing and positioning monitoring system is proposed in which passive RFID sticker tag is attached to patient's bracelet and medical personnel use PDA to scan RFID bracelet closely to verify patient's identification and location [25]. However, in our opinion, we think that using PDA manually to acquire patient's information stored on RFID tag is still considered manual work which may lead to possible human errors. Active RFID, on the other hand, can automatically detect patient's current location and identification. Standing from the patient's point of view, it is safer this way. Moreover, from the business point of view, issues like saving cost, environmental protection, and long-lasting usage always matter. Using active RFID to replace passive RFID is a better solution for business. In this paper, we proposed signal level matrix, SLM, to improve the accuracy and stability of active RFID indoor positioning system. A detailed description is discussed in later section.

\section{Methodology}

In the operating room, we place RFID reader at the entrance of each passageway to detect and transmit the message to the server when a RFID tag attached hospital bed passing through the reader. Reader refers to the signal strength of a tag to identify its location. However, with the same distance, the performance of the signal strength of tags is quite different that may influence the accuracy of the positioning system. In this paper, to solve the problem of overlapping sensing area and give consideration of the special environment characters of the operating room, a new framework that combines active RFID tags and signal level matrix, SLM, is proposed for the positioning and monitoring of surgical patients in the operating room. Construction of the system is described below.

3.1. Active RFID Positioning. In the RFID positioning system, tag and reader communicate wirelessly and the tag has builtin memory; it is divided into active or passive by the source of energy in the tag. The selection of active and passive RFID directly affects the design method, layout, positioning, and correction of the entire system. Therefore, the adoption of the active or passive RFID types is the primary consideration when constructing the system.

The active RFID covers a wider reading range and possesses distinguishable signal strength. The stronger the signal received, the closer the tag is to the reader, and vice versa. Thus, the target objects' whereabouts can be tracked. This feature can be used to calculate the locations of each surgical patient in order to accurately monitor the surgical process. It also aids in extending the preprocessing time for the various undertakings in order to be well prepared for the connection tasks. In addition, the active tag has a bigger memory data space and has the read and rewrite features which are useful in recording the surgical patients' identification. Furthermore, the active RFID can set the time interval for sending tag signals, thus achieving the effect of automatic sensing and reducing the back and forth audit process the medical and nursing personnel go through. Therefore, the active RFID tag is adopted by our proposed system.

3.2. The Framework of the Proposed Positioning System. The entire RFID system, including active tags, small power source readers, and an application system, has to be used with the antenna, in which each tag includes a designated number, a model number, place of assembly, and other data. The RFID middleware that we designed can safely send the RFID data read by the reader back to the server-end database. Its structure is as shown in Figure 1. Application program requests information of RFID tags from middleware, middleware via Ethernet requests information of RFID tags from the reader, the reader transmits electromagnetic waves to RFID tags, and then RFID tags passes information back to the reader. Since the tag's designated number is designed in binary data, middleware conduct data processing for received information and then these processed data will be sent back to the application program in the form of XML. 
Middleware sets up the relevant communication parameters such as Reader's IP address and Port. After the system issues the connection command, the successfulness of the connecting command is judged by the received data. After successful connection, the data that completed conversion are sent to the buffer to check if the serial number in the buffer is repeated. Data are saved in accordance with the XML document format, signed, and encrypted before being sent back to the server.

Within the changing environment, signal shielding caused by physical properties like reflection, diffraction, and refraction or interference by other electronic signals may influence signal strength which may lead to nonlinear proportional signal strength to distance and misjudgment on determining locations. To overcome the problem of interference, a positioning system usually includes positioning data record in the training database as shown in Figure 1. Based on the result of training database, abnormal signal strength caused by interference can be spotted and adjusted.

The best solution to solve the interferences from indoor static environmental attributes is to reinforce or weaken the signal strength of the tag affected by the interferences. However, for objects in the environment that cannot remain static over a long period of time, such as door opening and closing that instantly interfere with the RSS of the electronic devices, or the shielding effect produced by mobile individuals, we can use the signal weakening feature to judge whether an object exists or use the intuitive method to let the system automatically ignore extreme RSS values.

3.3. Distribution of Tags and Readers. Different from current distribution of readers which are mounted on the ceiling, we install reader near the gate and passageway at the position that is the same height with tags attached on hospital bed, shown in Figure 2, to reduce the interference caused by other objects within the hospital.

3.4. Signal Level Matrix (SLM). Received signal strength, RSS, is an important data for the RFID system to calculate the location of the tag. The weaker the RSS is, the more distant the range between reference point and target point is; meaning that RFID has the character of diminishing signal accompanied by the increase of distance between reader and tag. When moving a hospital bed with an RFID tag, the reader that is nearer to the tag can be used to locate the position of the tag. If multiple readers detect the existence of the same tag, readers have to determine the tag belonged section. The problem of overlapping sensing area occurred. This paper proposed the signal level matrix (SLM) to solve the multiple detecting problem caused by signal overlapping areas. The signal strength is classified into four levels according to the distance between reader and tag, shown in Table 1. And the signal level matrix of the area around a reader is set up according to the signal levels of the tags received by the reader and its neighboring reader. In Table $1, \mathrm{D}$ is as in detecting radius, meaning that the distance between two readers is $2 \mathrm{D}$ meters. In Table $1, \mathrm{~L}^{\dagger}{ }^{\dagger}$ represents the closest distance between reader and tag; $\mathrm{L}^{\Delta}$ represents a longer distance than $\mathrm{L}^{\dagger} ; \mathrm{L}^{\#}$
TABLE 1: Levels of signal strength.

\begin{tabular}{lc}
\hline Signal level & Distance between reader and tag \\
\hline $\mathrm{L}^{\dagger}$ & $<0.5 \mathrm{D}$ \\
$\mathrm{L}^{\Delta}$ & $0.5 \mathrm{D} \sim 1 \mathrm{D}$ \\
$\mathrm{L}^{\#}$ & $1 \mathrm{D} \sim 2 \mathrm{D}$ \\
$\mathrm{L}^{*}$ & $>2 \mathrm{D}$ \\
\hline
\end{tabular}

TABLE 2: The signal level matrix of a tag located in the area of A22.

\begin{tabular}{lll}
\hline $\mathrm{R} 11=\mathrm{L}^{*}$ & $\mathrm{R} 12=\mathrm{L} 3^{*}$ & $\mathrm{R} 13=\mathrm{L} 3^{*}$ \\
$\mathrm{R} 21=\mathrm{L}^{*}$ & $\mathrm{R} 22=\mathrm{L} 0^{\dagger}$ & $\mathrm{R} 23=\mathrm{L} 3^{*}$ \\
$\mathrm{R} 31=\mathrm{L}^{*}$ & $\mathrm{R} 32=\mathrm{L} 3^{*}$ & $\mathrm{R} 33=\mathrm{L} 3^{*}$ \\
\hline
\end{tabular}

represents the distance that is longer than $\mathrm{L1}^{\Delta}$; $\mathrm{L}^{*}$ represents the longest distance between reader and tag.

As shown in Figure 3, the entire area is divided into 9 sections. One Active RFID reader is located at the center of each section. The distance between two horizontally/vertically neighboring readers is $2 \mathrm{D}$ meters. Each reader is labeled with different number (R11, R12,..., and R33). There are two sensing ranges for each reader and their radius is D meters and $2 \mathrm{D}$ meters, respectively. Intersections occurred within the sensing range of the radius of $2 \mathrm{D}$ meters are illustrated as in Figure 3. To distinguish the location of tags, each section is further divided into subsections based on the distance between tag and reader. When a tag is very close to a reader within a range of $0.5 \mathrm{D}$ meter, this tag can be easily identified. When the distance between a tag and a reader is larger than $0.5 \mathrm{D}$ meter and less than 1 meter, this tag may appear in the ranges of A22U, A22D, A22L, A22R, A22UL, A22UR, A22DL, and A22DR. Noted that D is as in down; $U$ is as in up; $\mathrm{L}$ is as in left; and $\mathrm{R}$ is as in right. The position of the tag can be identified according to the relative distance between this tag and other reader close by.

Basically, based on the signal levels received by readers, matrix of signal levels received by readers can define a tag's location. If the signal level matrix is as shown in Table 2, this tag is definitely in the range of A22 because $\mathrm{R} 22$ receives the strongest signal coming from the tag. If the signal level matrix is as shown in Table 3, a tag's location is determined by the relative signal level received by neighboring readers. Each reader has 8 neighbors. If a tag is closer to one neighbor, that neighboring reader should receive a stronger signal than other neighboring readers. For example, as shown in Table 3(a), a neighboring reader of R22 named R12 receives a stronger signal, L2, than signal received by other neighbors, R11, R13, R21, R23, R31, R32, and R33; this tag is in the area of A22U. In Table 3, U means upper, D means down, L means left, and R means right. For instance, A22U means upper side of tag number 22.

\section{Simulation Model and Experimental Results}

4.1. A Simulation Model of the Proposed Positioning System. Surgery room has always been a busy place. To match the high standard required by the hospital, a simulation model was 


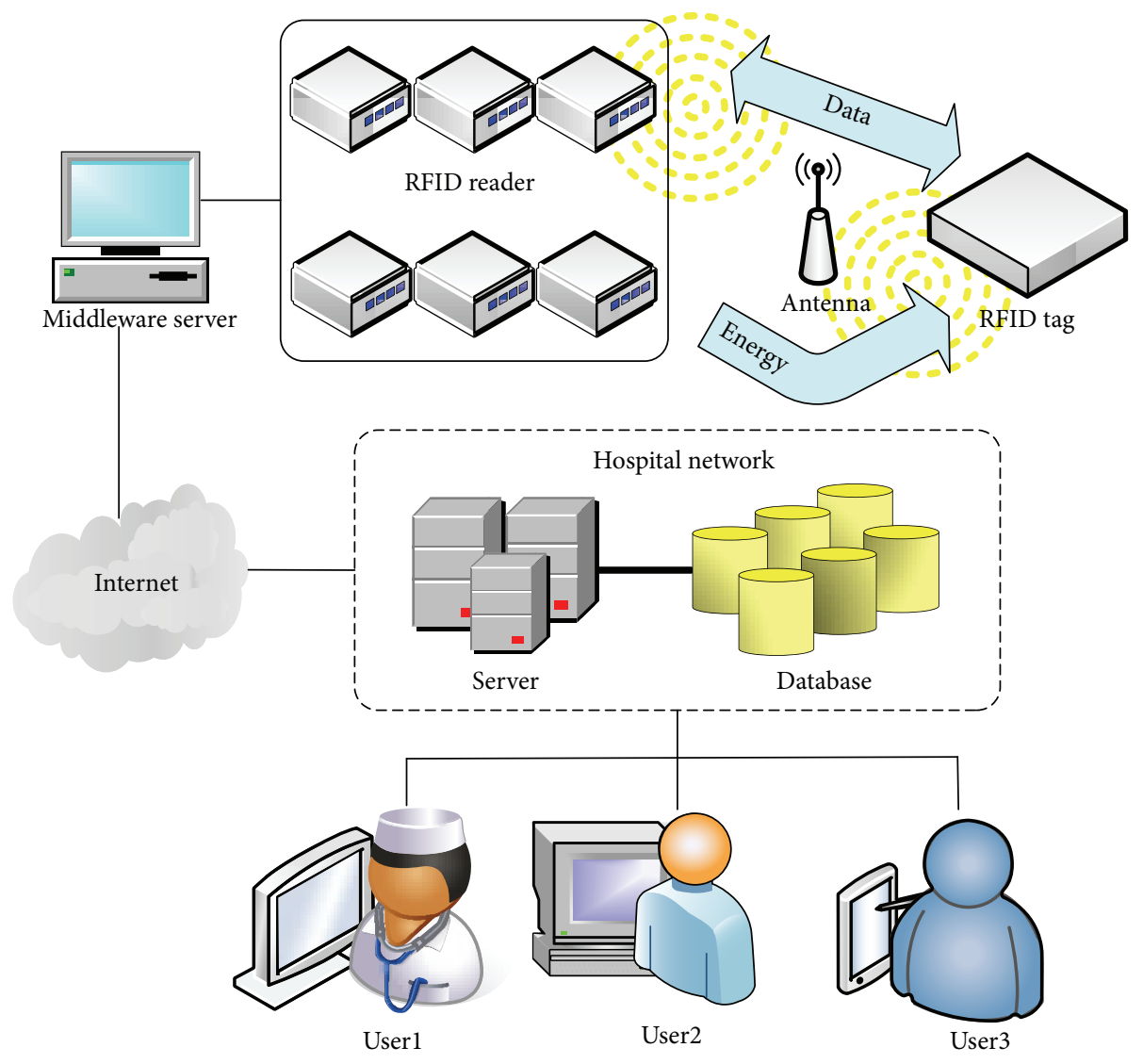

FIGURE 1: Functional component of the RFID System using in medical environment.

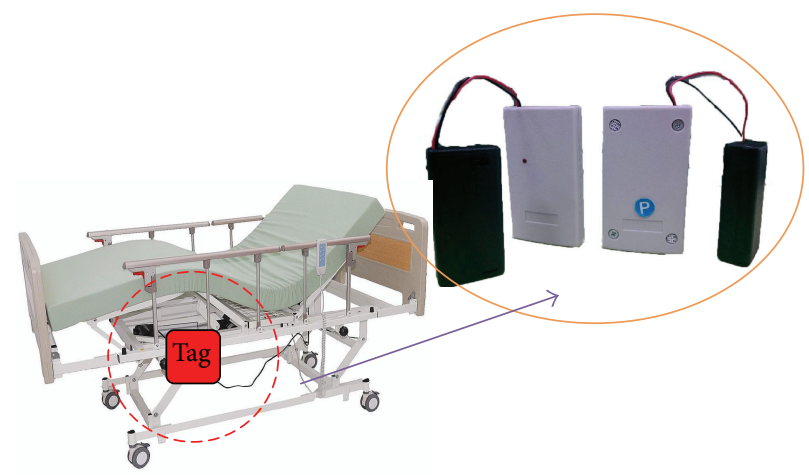

Figure 2: The placement of a tag on a hospital bed.

set up in a teaching hospital located in Taipei. As shown in Figure 4, the passageway is formed in $U$ shape in the surgery room based on the layout of the surgery room.

We use MRK-UMR40A RFID reader, UBM-T4ACT RFID tag, and MRK-UMP40A RFID repeater in the surgery room. A RFID repeater is used to transmit the ID number and RSSI to readers. The longest effective transmission distance between a repeater and a reader is 30 meters and the same distance between a repeater and a tag is 20 meters.

Repeater tag will send the tag signal strength received to the reader, so we can regard the reader as the server to receive the repeater's returned signal. After experiencing a number of practical measurements, we adjusted the signal strength of the tag and the distance between readers, to minimize the influence of signal interference.

Although the RSSI value of a RFID is not very stable, it is reversely proportional to the distance between a reader and a tag. To locate the position of hospital beds, the system can distinguish whether the hospital bed is inside or outside the surgery room by analyzing RSSI due to the segmentation effect caused by wall or door. Furthermore, due to the fact that the nature of the movement of hospital bed is continuous on 


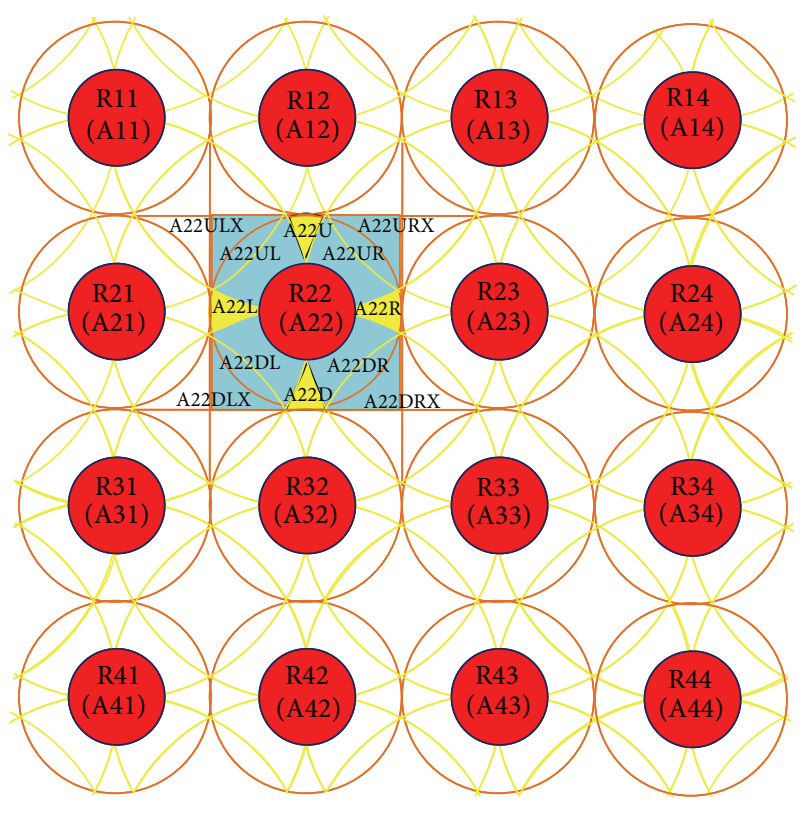

FIGURE 3: The schematic diagram of partitioned sections.

axis, the position of hospital bed can be determined based on the result of SLM.

4.2. Experimental Result. An experiment was conducted to determine the installation of the most cost effective RFID environment. In order to simulate the environment of surgery room, we use a $8 \mathrm{~m} \times 6 \mathrm{~m}$ lab room and a $30 \mathrm{~m} \times 2 \mathrm{~m}$ aisle outside the lab room. In spite of placing a RFID reader inside the lab room, several RFID repeaters were placed along the aisle outside the lab to transmit tag's information like ID number and RSSI values to the reader inside the lab room. Signals received by the reader are shown in Figure 5. There are three tags in this test. Tag 1 is the tag located inside the lab room. Tag 2 and tag 3 are located on the aisle. Among the signal strength sent by these three tags, signal sent by tag 1 can be received stably and its RF value is around -50 . Noted that the range between $\operatorname{tag} 1$ and the reader is around 1.5 meter, the distance between tag 2 and the reader is around 7 meters, and the distance between tag 3 and the reader is around 8 meters. Comparing with signal sent by the tag inside the lab room, signals sent by tags outside the lab room are relatively weak due to the interference caused by door or wall. Therefore, a threshold value is set to be -70 considering the factor of unstable transmission of signals caused by interference.

Besides detecting the signal strength received by the reader inside and outside the surgery room, we use a $30 \mathrm{~m}$ $\times 2 \mathrm{~m}$ aisle to simulate the environment outside the surgery room. Figure 6 shows the signal strength sent from a tag to the reader under different distance. The signal strength was measured at least one minute in each experiment. Although signal strength is not very stable during the experiment, basically, signal strength is negatively related to the distance between the reader and the tag within the range of 6 meters. Signal strength fluctuates severely when the distance between the reader and the tag is more than 6 meters. Therefore, signal
TABLE 3: The signal level matrix of a tag located in the areas of A22U, A22D, A22L, A22R, A22UL, A22UR, A22DL, and A22DR.

(a) Signal level matrix of A22U

\begin{tabular}{lll}
\hline $\mathrm{R} 11=\mathrm{L} 3^{*}$ & $\mathrm{R} 12=\mathrm{L} 2^{\#}$ & $\mathrm{R} 13=\mathrm{L} 3^{*}$ \\
$\mathrm{R} 21=\mathrm{L} 3^{*}$ & $\mathrm{R} 22=\mathrm{L} 1^{\Delta}$ & $\mathrm{R} 23=\mathrm{L} 3^{*}$ \\
$\mathrm{R} 31=\mathrm{L} 3^{*}$ & $\mathrm{R} 32=\mathrm{L} 3^{*}$ & $\mathrm{R} 33=\mathrm{L} 3^{*}$ \\
\hline
\end{tabular}

(b) Signal level matrix of A22D

\begin{tabular}{lll}
\hline $\mathrm{R} 11=\mathrm{L}^{*}$ & $\mathrm{R} 12=\mathrm{L} 3^{*}$ & $\mathrm{R} 13=\mathrm{L}^{*}$ \\
$\mathrm{R} 21=\mathrm{L}^{*}$ & $\mathrm{R} 22=\mathrm{L} 1^{\Delta}$ & $\mathrm{R} 23=\mathrm{L}^{*}$ \\
$\mathrm{R} 31=\mathrm{L} 3^{*}$ & $\mathrm{R} 32=\mathrm{L} 2^{\#}$ & $\mathrm{R} 33=\mathrm{L} 3^{*}$ \\
\hline
\end{tabular}

(c) Signal level matrix of A22L

\begin{tabular}{lll}
\hline $\mathrm{R} 11=\mathrm{L}^{*}$ & $\mathrm{R} 12=\mathrm{L} 3^{*}$ & $\mathrm{R} 13=\mathrm{L} 3^{*}$ \\
$\mathrm{R} 21=\mathrm{L}^{\#}$ & $\mathrm{R} 22=\mathrm{L} 1^{\Delta}$ & $\mathrm{R} 23=\mathrm{L} 3^{*}$ \\
$\mathrm{R} 31=\mathrm{L} 3^{*}$ & $\mathrm{R} 32=\mathrm{L} 3^{*}$ & $\mathrm{R} 33=\mathrm{L} 3^{*}$ \\
\hline
\end{tabular}

(d) Signal level matrix of A22R

\begin{tabular}{lll}
\hline $\mathrm{R} 11=\mathrm{L}^{*}$ & $\mathrm{R} 12=\mathrm{L}^{*}$ & $\mathrm{R} 13=\mathrm{L} 3^{*}$ \\
$\mathrm{R} 21=\mathrm{L}^{*}$ & $\mathrm{R} 22=\mathrm{L}^{\Delta}$ & $\mathrm{R} 23=\mathrm{L}^{*}$ \\
$\mathrm{R} 31=\mathrm{L} 3^{*}$ & $\mathrm{R} 32=\mathrm{L} 3^{*}$ & $\mathrm{R} 33=\mathrm{L} 3^{*}$ \\
\hline
\end{tabular}

(e) Signal level matrix of A22UL

\begin{tabular}{lll}
\hline $\mathrm{R} 11=\mathrm{L} 3^{*}$ & $\mathrm{R} 12=\mathrm{L} 2^{\#}$ & $\mathrm{R} 13=\mathrm{L}^{*}$ \\
$\mathrm{R} 21=\mathrm{L}^{*}$ & $\mathrm{R} 22=\mathrm{L}^{\Delta}$ & $\mathrm{R} 23=\mathrm{L}^{*}$ \\
$\mathrm{R} 31=\mathrm{L} 3^{*}$ & $\mathrm{R} 32=\mathrm{L}^{*}$ & $\mathrm{R} 33=\mathrm{L}^{*}$ \\
\hline
\end{tabular}

(f) Signal level matrix of A22UR

\begin{tabular}{lll}
\hline $\mathrm{R} 11=\mathrm{L}^{*}$ & $\mathrm{R} 12=\mathrm{L} 2^{\#}$ & $\mathrm{R} 13=\mathrm{L} 3^{*}$ \\
$\mathrm{R} 21=\mathrm{L} 3^{*}$ & $\mathrm{R} 22=\mathrm{L} 1^{\Delta}$ & $\mathrm{R} 23=\mathrm{L} 2^{\#}$ \\
$\mathrm{R} 31=\mathrm{L} 3^{*}$ & $\mathrm{R} 32=\mathrm{L} 3^{*}$ & $\mathrm{R} 33=\mathrm{L} 3^{*}$
\end{tabular}

(g) Signal level matrix of A22DL

\begin{tabular}{lll}
\hline $\mathrm{R} 11=\mathrm{L}^{*}$ & $\mathrm{R} 12=\mathrm{L} 3^{*}$ & $\mathrm{R} 13=\mathrm{L}^{*}$ \\
$\mathrm{R} 21=\mathrm{L} 2^{\#}$ & $\mathrm{R} 22=\mathrm{L} 1^{\Delta}$ & $\mathrm{R} 23=\mathrm{L}^{*}$ \\
$\mathrm{R} 31=\mathrm{L} 3^{*}$ & $\mathrm{R} 32=\mathrm{L} 2^{\#}$ & $\mathrm{R} 33=\mathrm{L} 3^{*}$
\end{tabular}

(h) Signal level matrix of A22DR

\begin{tabular}{lll}
\hline $\mathrm{R} 11=\mathrm{L}^{*}$ & $\mathrm{R} 12=\mathrm{L} 3^{*}$ & $\mathrm{R} 13=\mathrm{L} 3^{*}$ \\
$\mathrm{R} 21=\mathrm{L}^{*}$ & $\mathrm{R} 22=\mathrm{L}^{\Delta}$ & $\mathrm{R} 23=\mathrm{L}^{*}$ \\
$\mathrm{R} 31=\mathrm{L} 3^{*}$ & $\mathrm{R} 32=\mathrm{L} 2^{*}$ & $\mathrm{R} 33=\mathrm{L} 3^{*}$ \\
\hline
\end{tabular}

strength received within the range of 6 meters is reliable and constant. The average value of signal strength which was sent by three different tags and was measured in different ranges is illustrated in Figure 7. As shown in Figure 7, we can establish appropriate signal strength matrix based on the average signal strength received within different ranges.

Theoretically, RFID locating system has the following rule. The more intensive the distribution of readers is, the higher the degree of accuracy is. However, while enjoying higher degree of accuracy, the cost of installing RFID system increases and the problem of signal collision arises. Thus, to consider the factor of accuracy and costs, we conducted several experiments to determine the most cost effective method with the higher degree of accuracy. Table 4 shows the 


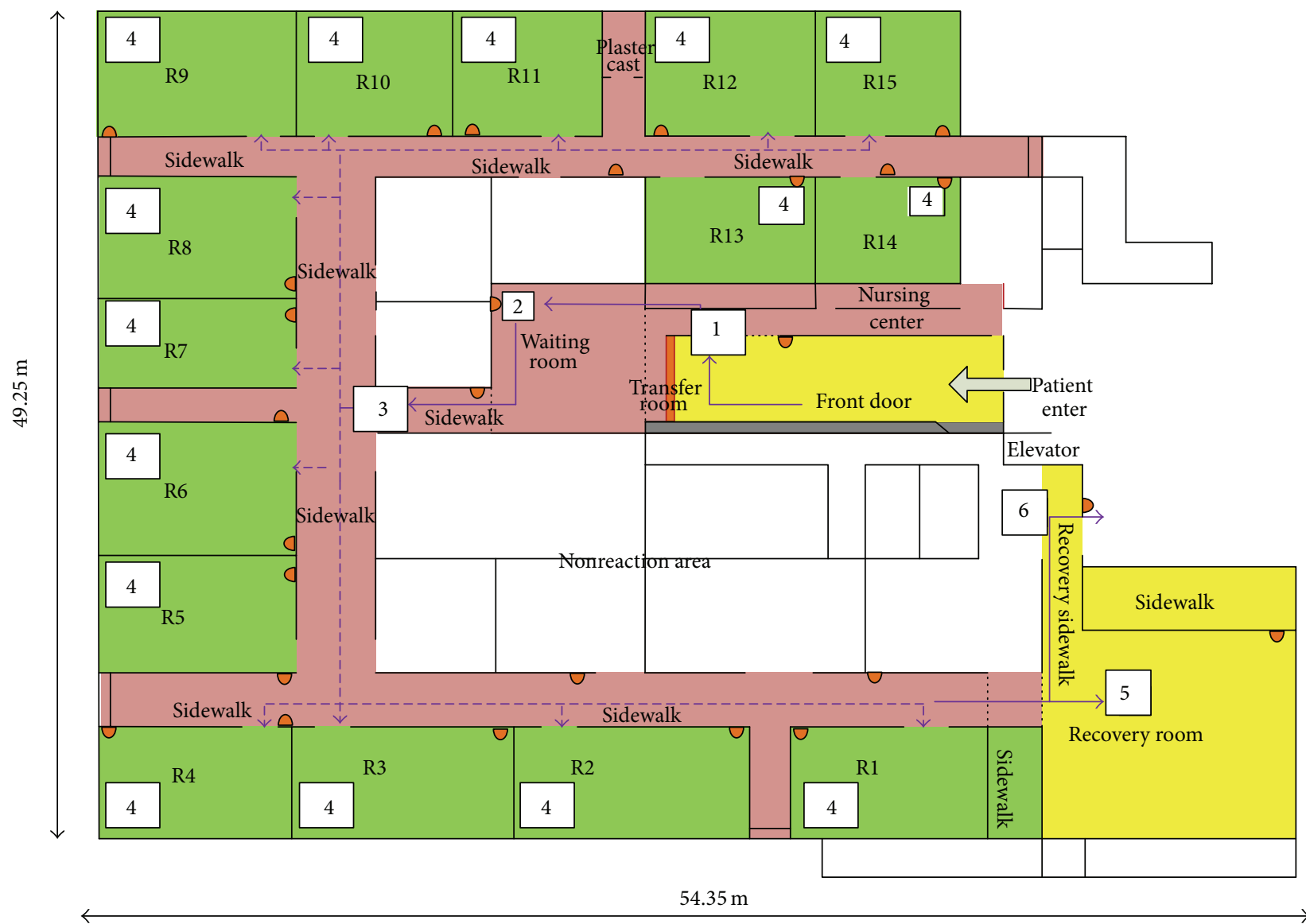

RFID reader

\begin{tabular}{|c|c|}
\hline Step & Location \\
\hline$(1)$ & Enter the front door \\
\hline$(2)$ & Stay in the waiting room \\
\hline$(3)$ & Go through the sidewalk \\
\hline$(4)$ & Enter the OP room \\
\hline$(5)$ & Stay in the recovery room \\
\hline$(6)$ & Leave the surgical area \\
\hline
\end{tabular}

FIgURE 4: The layout of the surgery room of a teaching hospital located in Taipei, Taiwan.

signal strength matrix under different distances between two readers.

The degree of accuracy of the experiment conducted under different distance between two readers (D) is listed in Table 5. The degree of accuracy is between $80 \%$ and $100 \%$ if $\mathrm{D}$ equals 2 and the average value of the degree of accuracy is $94 \%$. The degree of accuracy is between $90 \%$ and $100 \%$ if $\mathrm{D}$ equals 4 . The average value of the degree of accuracy is $98 \%$ and it the highest value among all tests. The degree of accuracy is between $85 \%$ and $100 \%$ if D equals 6 and the average value of the degree of accuracy is $97 \%$. The degree of accuracy is between $85 \%$ and $100 \%$ if $\mathrm{D}$ equals 8 and the average value of the degree of accuracy is $93 \%$. The degree of accuracy decreases dramatically when the distance between two readers is 12 meters and the average value of the degree of accuracy is only $69 \%$. Based on the finding of the experiment, the degree of accuracy can be above $90 \%$ if $\mathrm{D}$ is less than
8 meters (including 8 meters). Since the degree of accuracy drops to $69 \%$, we do not consider 12 meters as an option.

Therefore, we simulate the installation of RFID reader/repeater in the surgery room with different distances between two readers. The amount of RFID readers used in setting up the RFID environment under different distance between two readers is shown in Table 6 . Since 12 meters is not an option, we only consider the situation where $\mathrm{D}$ is less than 8 meters. According to this table, the more intensive the distribution of RFID readers is, the more the amount of RFID readers is used. When the distance between two readers is 2 meters, number of readers used is almost doubled than the number of readers used when the same distance is 6 meters. Also, when the distance between two readers is 2 meters, the degree of accuracy is less the degree of accuracy when the same distance is 6 meters. Thus, when the distance between two readers is 2 meters, it is not a satisfactory choice. 


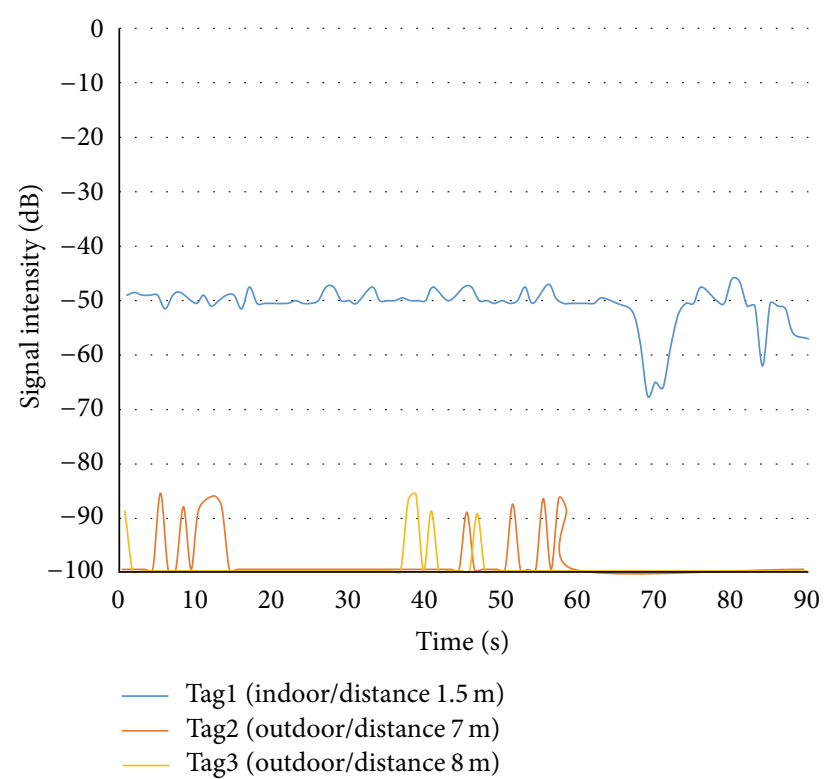

Figure 5: Signal Strength received by the reader placed inside the lab room.

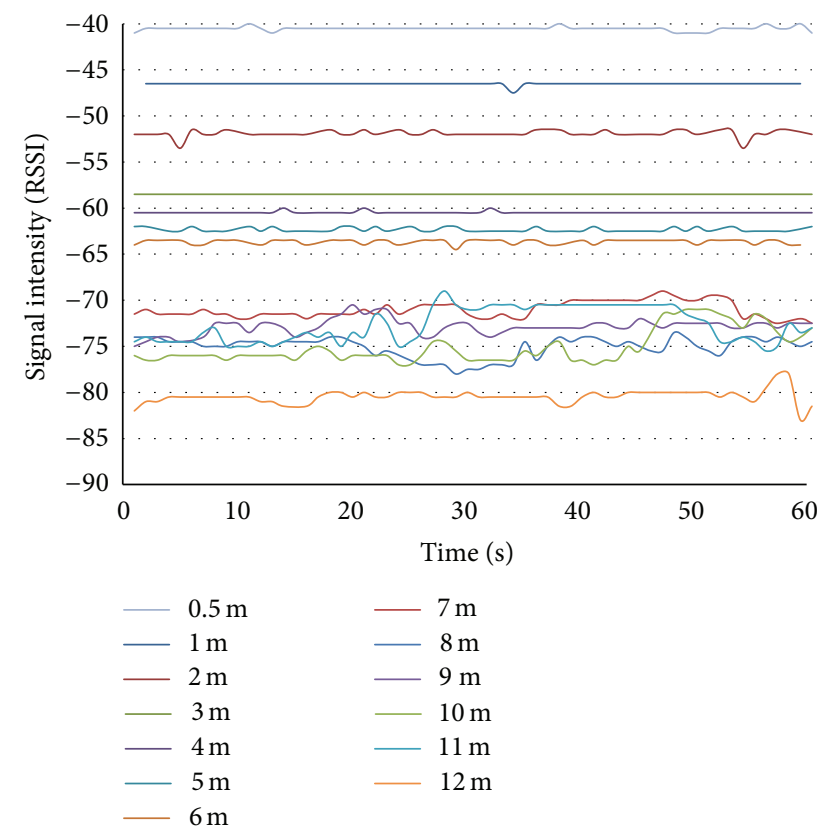

FIGURE 6: Signal strength measured within different ranges.

Furthermore, comparing the cases of 4 meters and 6 meters, the difference between their degrees of accuracy is only one percent while the number of readers used in 6-meters case is less by 8 readers than the 8 -meters case. When the issue of costs is considered in setting up the environment, it seems that 6 meters and 8 meters are both acceptable.

A simulated installation of RFID readers with 6-meters distance between two readers in the surgery room is illustrated in Figure 8. In the physical layout, we configured readers, respectively in the front room, the waiting area,
TABLE 4: The signal strength matrix under different distances between two readers.

(a) Distance between two readers is 2 meters

\begin{tabular}{lcc}
\hline Signal level & Distance between tag and reader & RSS \\
\hline $0^{\dagger}$ & $<0.5 \mathrm{~m}$ & $>-38.5$ \\
$\mathrm{L1}^{\Delta}$ & $0.5 \mathrm{~m} \sim 1 \mathrm{~m}$ & $-39 \sim-47.5$ \\
$\mathrm{~L}^{\#}$ & $1 \mathrm{~m} \sim 2 \mathrm{~m}$ & $-48 \sim-52.5$ \\
$\mathrm{~L}^{*}$ & $2 \mathrm{~m} \sim 3 \mathrm{~m}$ & $-53 \sim-65$ \\
\hline
\end{tabular}

(b) Distance between two readers is 4 meters

\begin{tabular}{lcc}
\hline Signal level & Distance between tag and reader & RSS \\
\hline $\mathrm{L}^{\dagger}$ & $<1 \mathrm{~m}$ & $>-46$ \\
$\mathrm{~L}^{\Delta}$ & $1 \mathrm{~m} \sim 2 \mathrm{~m}$ & $-46.5 \sim-53.5$ \\
$\mathrm{~L}^{\#}$ & $2 \mathrm{~m} \sim 4 \mathrm{~m}$ & $-54 \sim-59.5$ \\
$\mathrm{~L}^{*}$ & $4 \mathrm{~m} \sim 6 \mathrm{~m}$ & $-60 \sim-67$ \\
\hline
\end{tabular}

(c) Distance between two readers is 6 meters

\begin{tabular}{lcc}
\hline Signal level & Distance between tag and reader & RSS \\
\hline $\mathrm{L}^{\dagger}$ & $<1 \mathrm{~m}$ & $>-46$ \\
$\mathrm{L1}^{\Delta}$ & $1 \mathrm{~m} \sim 3 \mathrm{~m}$ & $-46.5 \sim-58$ \\
$\mathrm{~L}^{\#}$ & $3 \mathrm{~m} \sim 6 \mathrm{~m}$ & $-58.5 \sim-64$ \\
$\mathrm{~L}^{*}$ & $6 \mathrm{~m} \sim 9 \mathrm{~m}$ & $-64 \sim-77$ \\
\hline
\end{tabular}

(d) Distance between two readers is 8 meters

\begin{tabular}{lcc}
\hline Signal level & Distance between tag and reader & RSS \\
\hline $\mathrm{L}^{\dagger}$ & $<1 \mathrm{~m}$ & $>-46$ \\
$\mathrm{~L}^{\Delta}$ & $1 \mathrm{~m} \sim 4 \mathrm{~m}$ & $-46.5 \sim-59.5$ \\
$\mathrm{~L}^{\#}$ & $4 \mathrm{~m} \sim 8 \mathrm{~m}$ & $-60 \sim-69.5$ \\
$\mathrm{~L}^{*}$ & $8 \mathrm{~m} \sim 12 \mathrm{~m}$ & $-70 \sim-84$ \\
\hline
\end{tabular}

(e) Distance between two readers is 12 meters

\begin{tabular}{lcc}
\hline Signal level & Distance between tag and reader & RSS \\
\hline $\mathrm{L}^{\dagger}$ & $<1 \mathrm{~m}$ & $>-46$ \\
$\mathrm{~L}^{\Delta}$ & $1 \mathrm{~m} \sim 6 \mathrm{~m}$ & $-46.5 \sim-67.5$ \\
$\mathrm{~L}^{\#}$ & $6 \mathrm{~m} \sim 12 \mathrm{~m}$ & $-68 \sim-73.5$ \\
$\mathrm{~L}^{*}$ & $12 \mathrm{~m} \sim 18 \mathrm{~m}$ & $-74 \sim-90$ \\
\hline
\end{tabular}

the recovery room, and the operating rooms. The operating rooms are segmented by walls and metal doors. Signals in the operating rooms do not interfere with others. On the pathway, in order to avoid the signal exceeding the receiving range and resulting in the loss of signals, readers are placed on the wall along the pathway. Therefore, as shown in Figure 8, along the pathway, there are at least 1 to 2 readers being able to read the tag signal.

\section{Conclusion}

This paper presents a system that can identify the location of a surgical patient in an operation room based on the different phases in the process of a surgery. In this system, RFID technology that uses active RFID tags and places readers in the environment of an operation room is adopted to monitor and locate the position of surgical patients prior to, in the middle, and after a surgery. The proposed signal 


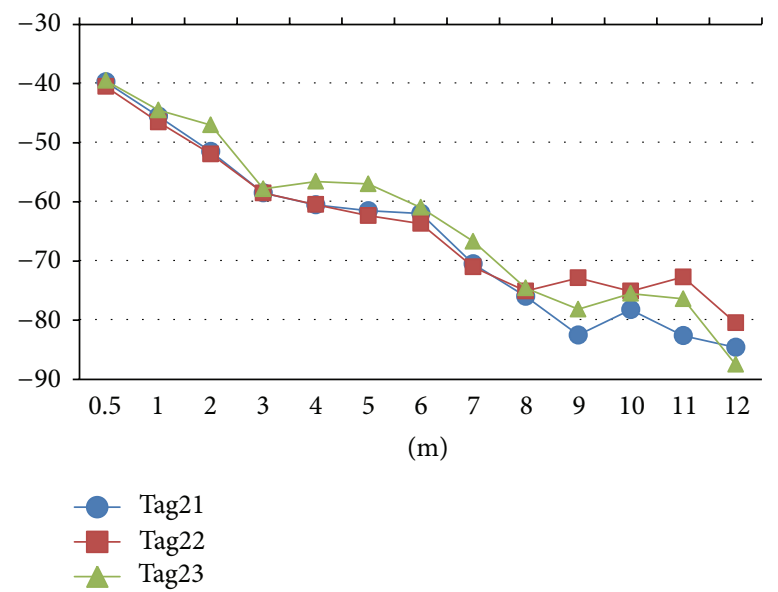

FIGURE 7: Average value of signal strength sent by three tags and measured within different ranges.

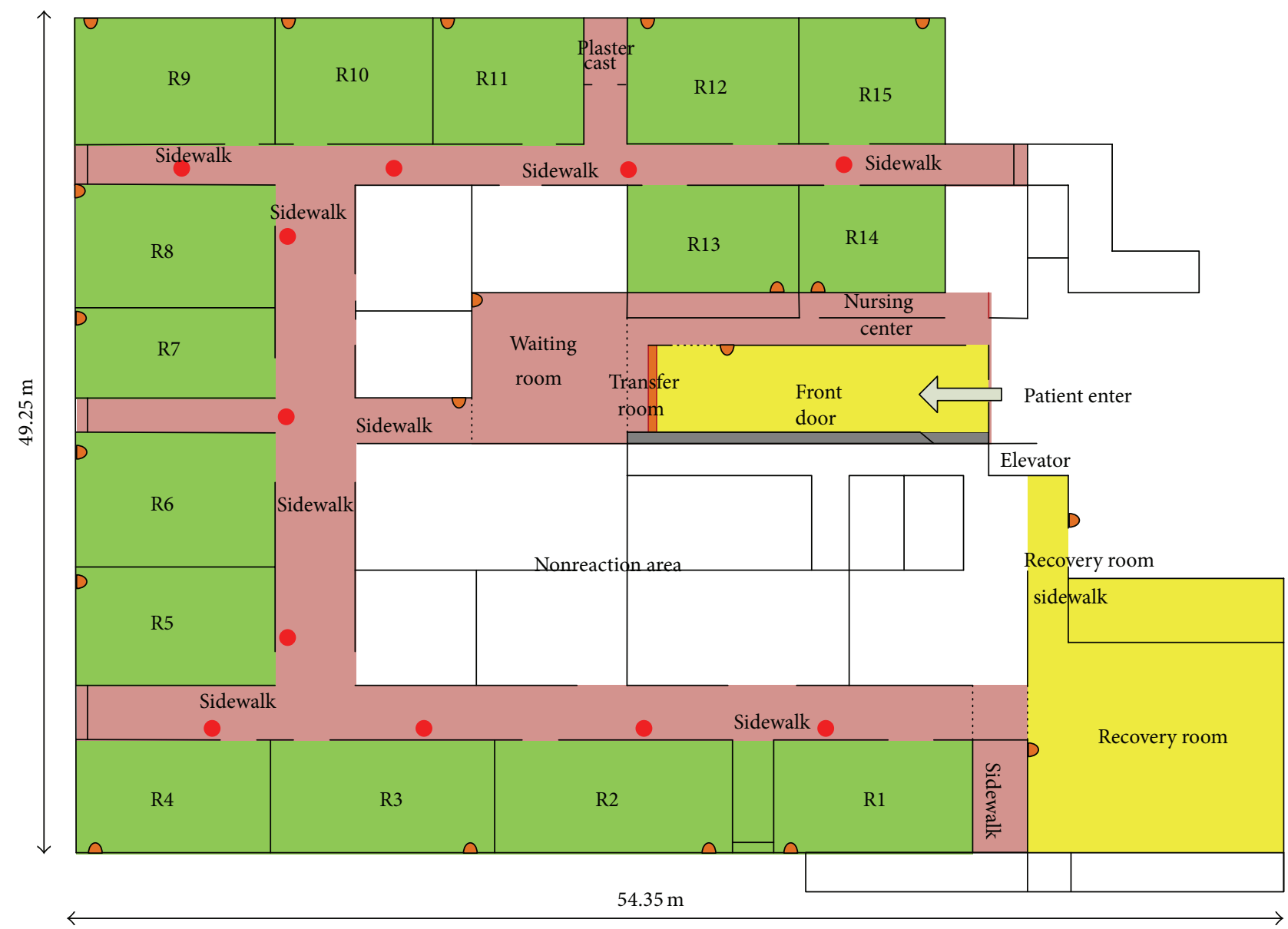

RFID reader

Sidewalk reader

FIGURE 8: A simulated installation of RFID readers with 6-meters distance between two readers in the surgery room. 
TABLE 5: The degree of accuracy of detecting the location of tags in divided areas under different distances between two readers.

\begin{tabular}{|c|c|c|c|c|c|c|c|}
\hline \multirow{2}{*}{ Distance between two readers } & \multicolumn{7}{|c|}{ Divided areas } \\
\hline & A22 & $\mathrm{A} 22 \mathrm{U}$ & $\mathrm{A} 22 \mathrm{D}$ & A22UL & A22DR & A22DRX & Average \\
\hline 2 meters & $100 \%$ & $100 \%$ & $80 \%$ & $85 \%$ & $100 \%$ & $100 \%$ & $94 \%$ \\
\hline 4 meters & $100 \%$ & $100 \%$ & $100 \%$ & $90 \%$ & $100 \%$ & $100 \%$ & $98 \%$ \\
\hline 6 meters & $100 \%$ & $95 \%$ & $100 \%$ & $100 \%$ & $100 \%$ & $85 \%$ & $97 \%$ \\
\hline 8 meters & $100 \%$ & $95 \%$ & $90 \%$ & $85 \%$ & $85 \%$ & $100 \%$ & $93 \%$ \\
\hline 12 meters & $100 \%$ & $85 \%$ & $100 \%$ & $25 \%$ & $25 \%$ & $80 \%$ & $69 \%$ \\
\hline
\end{tabular}

TABLE 6: Number of tags used under different distance between two readers.

\begin{tabular}{|c|c|c|c|}
\hline \multirow{2}{*}{ Distance between two readers } & \multicolumn{3}{|c|}{ Number of tags used } \\
\hline & Number of readers used indoor & Number of readers used outdoor & Total number of readers used \\
\hline 2 meters & \multirow{4}{*}{20} & 44 & 64 \\
\hline 4 meters & & 22 & 42 \\
\hline 6 meters & & 14 & 34 \\
\hline 8 meters & & 11 & 31 \\
\hline
\end{tabular}

level matrix is to solve the problem of sensing overlapping area based on the level of signal strength sent by tags. While moving a hospital bed, there are two kinds of characters: signal strength is in reverse proportion to distance and the movement of a hospital bed is a continuous function shown on a coordinate. These two characters are used to find the location of a hospital bed. A simulation is conducted to imitate the actual situation that occurred in a surgery room. The experiment result shows that this system can accurately identify surgical patient's identification and the position of this patient. By constantly updating patients' location, all relevant units receive correct and newest message about a patient's current status and location to coordinate the work of taking care of a surgical patient smoothly. On one hand, this system can avoid irreparable negligence caused by human error or delayed notification. On the other hand, health care personnel can concentrate more on performing the surgery to provide a higher quality of medical care.

\section{Conflict of Interests}

The authors declare that there is no conflict of interests regarding the publication of this paper.

\section{Acknowledgments}

This research is supported by National Science Council of Taiwan, under research Project NSC102-2221-E-227-001. Also, special thanks are due to Mr. Shen-Yuan Tang, the director of the department of Information Management in Shin Kong Wu Ho-Su Memorial Hospital, for his consultation and suggestion on this project.

\section{References}

[1] L. M. Ni, D. Zhang, and M. R. Souryal, "RFID-based localization and tracking technologies," IEEE Wireless Communications, vol. 18, no. 2, pp. 45-51, 2011.
[2] A. Grover and H. Berghel, "A survey of RFID deployment and security issues," Journal of Information Processing Systems, vol. 7, no. 4, pp. 561-580, 2011.

[3] D. M. Konidala, D. Kim, C. Y. Yeun, and B. Lee, "Security framework for RFID-based applications in smart home environment," Journal of Information Processing Systems, vol. 7, no. 1, pp. 111120, 2011.

[4] J. Ahn and R. Han, "An indoor augmented-reality evacuation system for the Smartphone using personalized Pedometry," Human-Centric Computing and Information Sciences, vol. 2, pp. 1-23, 2012.

[5] J. Chen, M. B. Salim, and M. Matsumoto, "A single mobile target tracking in voronoi-based clustered wireless sensor network," Journal of Information Processing Systems, vol. 7, pp. 17-28, 2011.

[6] Y. Luo, O. Hoeber, and Y. Chen, "Enhancing Wi-Fi fingerprinting for indoor positioning using human-centric collaborative feedback," Human-Centric Computing and Information Sciences, vol. 3, article 2, 2013.

[7] S. S. Saad and Z. S. Nakad, "A standalone RFID indoor positioning system using passive tags," IEEE Transactions on Industrial Electronics, vol. 58, no. 5, pp. 1961-1970, 2011.

[8] R. Want, "An introduction to RFID technology," IEEE Pervasive Computing, vol. 5, no. 1, pp. 25-33, 2006.

[9] J. Hightower, R. Want, and G. Borriello, SpotON: An Indoor 3D Location Sensing Technology Based on RF Signal Strength, 2000.

[10] M. N. Lionel, Y. Liu, Y. C. Lau, and A. P. Patil, "LANDMARC: indoor location sensing using active RFID," Wireless Networks, vol. 10, no. 6, pp. 701-710, 2004.

[11] Y. Zhao, Y. Liu, and L. M. Ni, "VIRE: active RFID-based localization using virtual reference elimination," in Proceedings of the 36th International Conference on Parallel Processing (ICPP '07), p. 56, Xi'an, China, September 2007.

[12] P. Krishnan, A. S. Krishnakumar, W. Ju, C. Mallows, and S. Ganu, "A system for LEASE: location estimation assisted by stationary emitters for indoor RF wireless networks," in Proceedings of the 23rd Annual Joint Conference of the IEEE Computer and Communications Societies (INFOCOM '04), pp. 1001-1011, Hong Kong, March 2004.

[13] C.-S. Wang, X.-M. Huang, and M.-Y. Hung, "Adaptive rfid positioning system using signal level matrix," in Proceedings of 
the International Conference on Sensor Networks, Information, and Ubiquitous Computing, Singapore, 2010.

[14] R. Hosaka, "Feasibility study of convenient automatic identification system of medical articles using LF-band RFID in hospital," Systems and Computers in Japan, vol. 35, no. 10, pp. 74-82, 2004.

[15] D.-H. Shih, H.-S. Chiang, B. Lin, and S.-B. Lin, "An embedded mobile ECG reasoning system for elderly patients," IEEE Transactions on Information Technology in Biomedicine, vol. 14, no. 3, pp. 854-865, 2010.

[16] R. Want, A. Hopper, V. Falcao, and J. Gibbons, "The active badge location system," ACM Transactions on Information Systems, vol. 10, no. 1, pp. 91-102, 1992.

[17] C. A. Thompson, "Radio frequency tags for identifying legitimate drug products discussed by tech industry," American Journal of Health-System Pharmacy, vol. 61, no. 14, pp. 14301432, 2004.

[18] J. A. Fisher and T. Monahan, "Tracking the social dimensions of RFID systems in hospitals," International Journal of Medical Informatics, vol. 77, no. 3, pp. 176-183, 2008.

[19] Y. Gu, A. Lo, and I. Niemegeers, "A survey of indoor positioning systems for wireless personal networks," IEEE Communications Surveys \& Tutorials, vol. 11, no. 1, pp. 13-32, 2009.

[20] I. D'Souza, W. Ma, and C. Notobartolo, "Real-time location systems for hospital emergency response," IT Professional, vol. 13, no. 2, pp. 37-43, 2011.

[21] G. B. Gentili, F. Dori, and E. Iadanza, "Dual-frequency active RFID solution for tracking patients in a children's hospital. Design method, test procedure, risk analysis, and technical solution," Proceedings of the IEEE, vol. 98, no. 9, pp. 1656-1662, 2010.

[22] J. K.-Y. Ng, "Ubiquitous healthcare: healthcare systems and applications enabled by mobile and wireless technologies," Journal of Convergence, vol. 3, pp. 15-20, 2012.

[23] D. Kang, K. Kang, H. Lee, E. Ko, and J. Lee, "A systematic design tool of context aware system for ubiquitous healthcare service in a smart home," in Proceedings of the International Conference on Future Generation Communication and Networking (FGCN '07), pp. 49-54, Jeju, Republic of Korea, December 2007.

[24] J. E. Bardram and N. Nørskov, "A context-aware patient safety system for the operating room," in Proceedings of the 10th International Conference on Ubiquitous Computing (UbiComp '08), pp. 272-281, September 2008.

[25] R. Tesoriero, J. A. Gallud, M. Lozano, and V. M. R. Penichet, "Using active and passive RFID technology to support indoor location-aware systems," IEEE Transactions on Consumer Electronics, vol. 54, no. 2, pp. 578-583, 2008. 


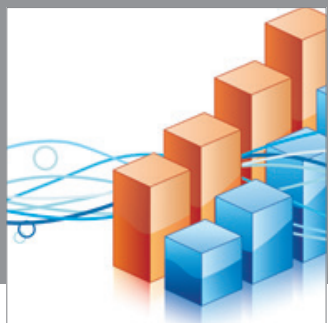

Advances in

Operations Research

mansans

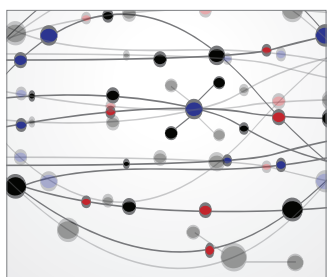

The Scientific World Journal
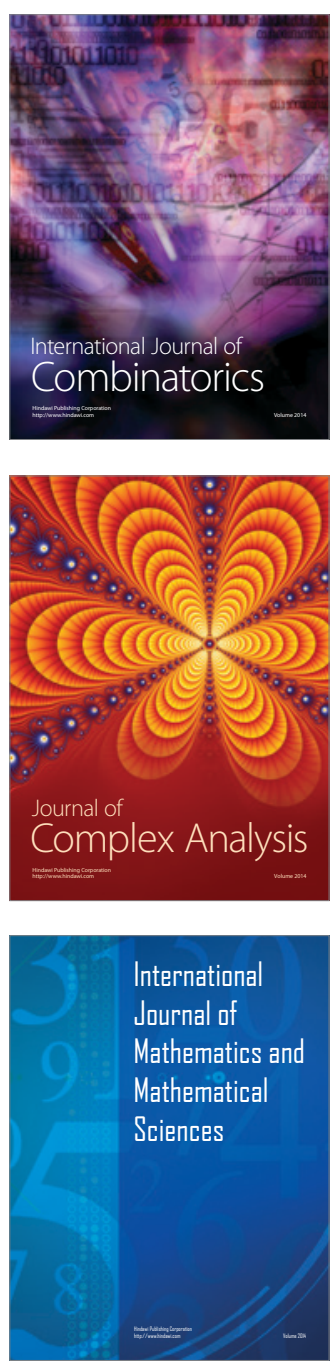
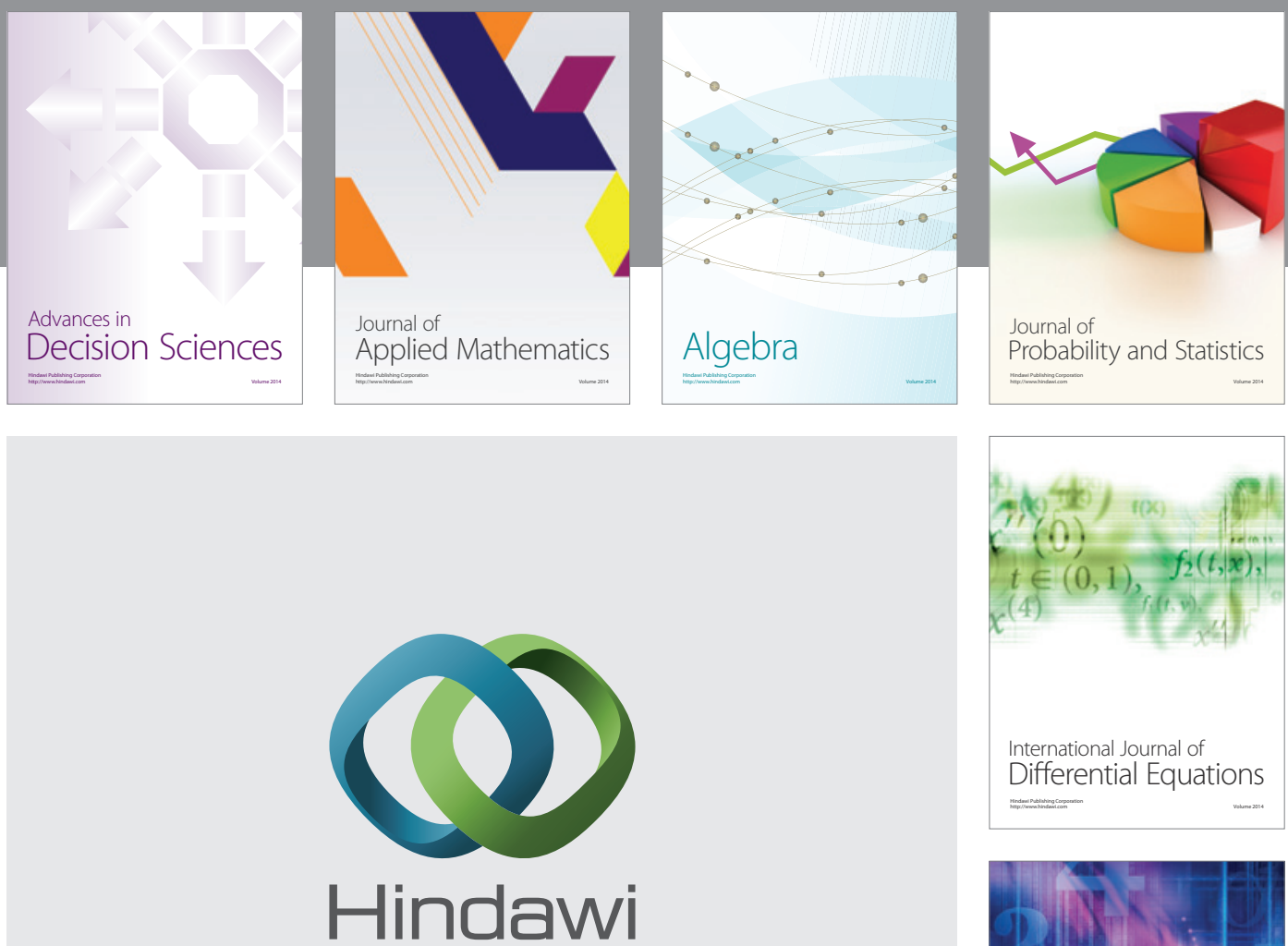

Submit your manuscripts at http://www.hindawi.com
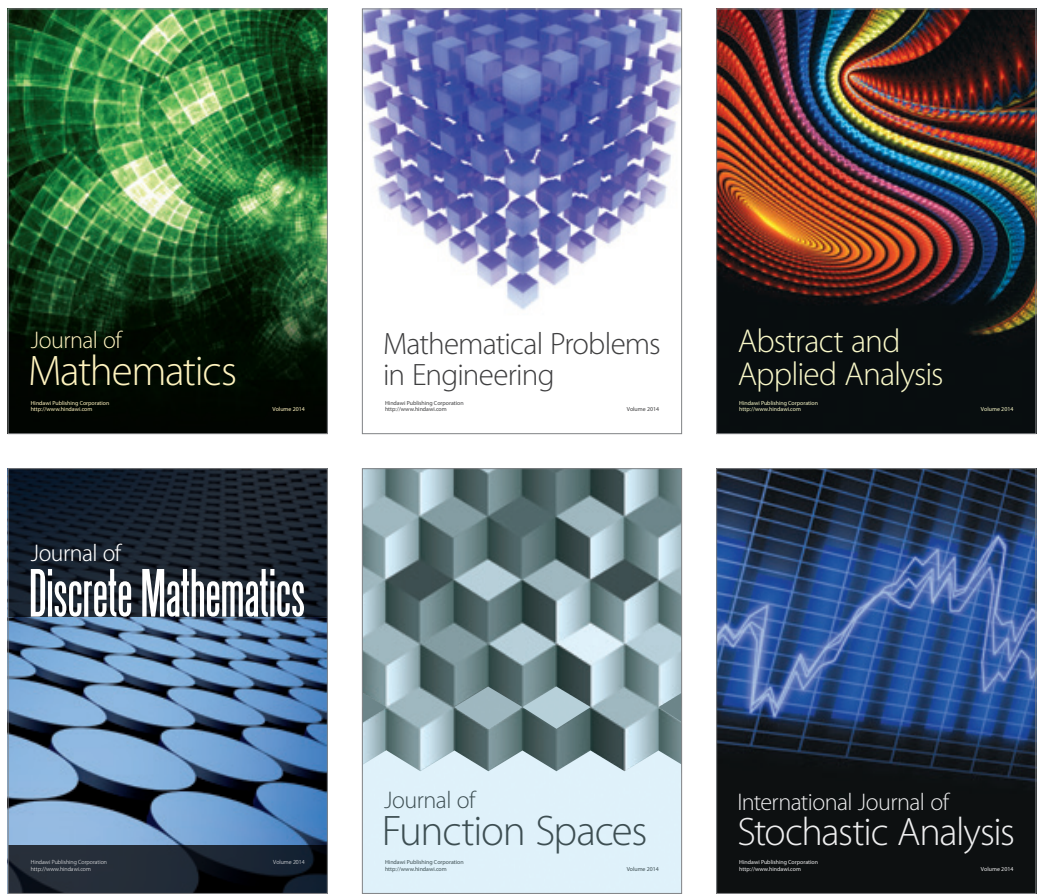

Journal of

Function Spaces

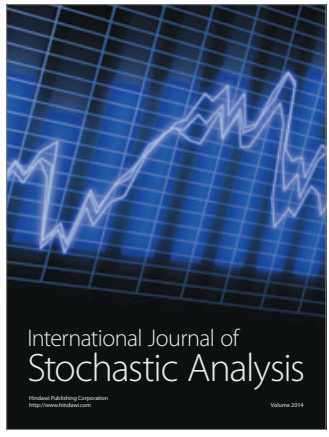

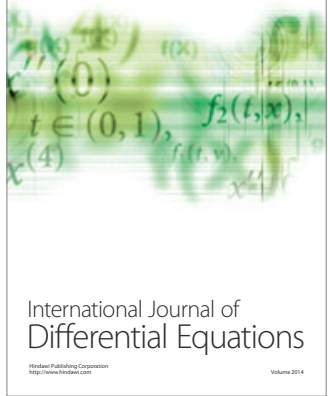
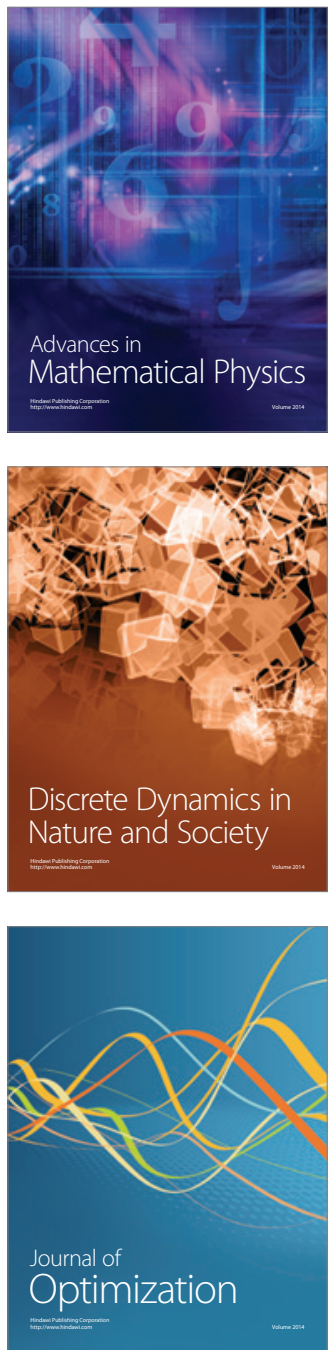